\title{
Permanence of a General Periodic Single-Species System with Periodic Impulsive Perturbations
}

\author{
Xianning $\operatorname{LiU}^{a, b, *}$ and Yasuhiro TAKEUCHI ${ }^{b}$ \\ ${ }^{a}$ Key Laboratory of Eco-environments in Three Gorges Reservoir Region \\ (Ministry of Education) \\ School of Mathematics and Statistics, Southwest University \\ Chongqing, 400715, P.R. China \\ E-mail: liuxn@swu.edu.cn \\ ${ }^{b}$ Department of Systems Engineering, Shizuoka University \\ Hamamatsu, 432-8561, Japan
}

Received August 9, 2006

Revised September 21, 2006

Sufficient conditions for permanence of a general periodic single-species system with periodic impulsive perturbations are obtained via comparison theory of impulsive differential equations. An application is given to the periodic impulsive logistic system.

Key words: impulses, single-species, permanence, periodic perturbation

\section{Introduction}

Impulsive phenomena appear widely in the real world. For example, in fishery, human actions on fish resources (harvesting or planting) are seasonal or occur in regular pulses; To finish a vaccination process of disease (such as Hepatitis B etc.), doses usually should be taken several times and there must be some fixed time intervals between two doses; People may use the pesticides to kill the pest at some certain moments instead of using it continuously; Chemical treatment of disease cannot be done continuously since it destroys both infected and healthy cells, and so on. To study the dynamics of such kind of processes, it is important to consider these perturbations impulsively. Impulsive differential equations are suitable for the mathematical simulations of such evolutionary processes whose states are subject to sudden changes at certain moments. Recently, there has been some investigations considering realistic impulsive effects in population dynamics, such as inputs of substrates [2], birth [3, 11, 13], chemotherapeutic treatments [4], pest control [8] or planting and harvesting of species [9] and vaccinations against disease [12] etc. Usually, it is difficult to analyze the impulsive differential equations arisen from applications due to numerous theoretical and technical difficulties except that in some cases the models can be rewritten as simple discrete-time mapping or difference equations when the corresponding continuous models can be solved explicitly, eg. $[2,13]$. This is the reason that numeric simulations are frequently used in applications. Recently, many investigations focus on the global dynamics of impulsive

\footnotetext{
* Supported by the National Natural Science Foundation of China (10571143), the Science Foundation of Southwest University (SWNUB2004001) and the Japanese Government (Monbukagakusho: MEXT) Scholarship.
} 
systems, see, for example $[6,9,10,14,16,17]$.

In this paper, we will consider the following nonlinear impulsive system in $R_{+}$.

$$
\begin{gathered}
x^{\prime}(t)=x(t) f(t, x(t)), \quad t \neq \tau_{k}, \quad k \in N, \\
\triangle x\left(\tau_{k}\right)=b_{k} x\left(\tau_{k}\right), \quad k \in N,
\end{gathered}
$$

where $N$ is the set of positive integers, $\tau_{0} \triangleq 0<\tau_{1}<\cdots<\tau_{k}<\tau_{k+1}<\cdots$, $\triangle x\left(\tau_{k}\right)=x\left(\tau_{k}^{+}\right)-x\left(\tau_{k}\right)$. (1.1) may describe the variation of population number of an isolated species, for example, some kind of fish species. (1.2) describes the human exploit activities which are considered as impulsive perturbations of the species since harvesting or planting of species is actually seasonal or occurs in regular pulses.

Suppose that system (1.1), (1.2) satisfies the following conditions (A1)-(A4).

(A1) (1.1) is $\omega$-periodic and (1.2) is $T$-periodic, i.e.,

$$
f(t+\omega, x) \equiv f(t, x), \quad t \in R,
$$

$T$ is the least positive constant such that there are $l \tau_{k}$ s in the interval $(0, T)$ and

$$
\tau_{k+l}=\tau_{k}+T, \quad b_{k+l}=b_{k}, \quad k \in N .
$$

(A2) $\quad f(\cdot, x) \in P C[R, R]$ and $P C[R, R]=\{\phi: R \mapsto R, \phi$ is continuous for $t \neq$ $\tau_{k}, \phi\left(\tau_{k}^{+}\right)$and $\phi\left(\tau_{k}^{-}\right)$exist and $\left.\phi\left(\tau_{k}\right)=\phi\left(\tau_{k}^{-}\right), k \in N\right\}$.

(A3) $f(t, \cdot)$ is differentiable and $\partial f / \partial x \leq 0$.

(A4) $1+b_{k}>0, b_{k} \neq 0, k \in N$.

(1.3) in (A1) shows that the variation of the population number $x(t)$ is $\omega$-periodic, which may be related to the periodically changing of environment. This can describe the periodically changing possibility of regeneration of the species and the periodic changing of the resources maintaining the evolution of the population. (1.4) shows that impulsive effects on the population are $T$-periodic. Naturally, this period is distinct from $\omega$, the period of the change of environment. Even when we want to carry out the perturbations according to the period $\omega$, we cannot do it since we do not know $\omega$ exactly. (A3) shows the species is density dependent. When $b_{k}>0$, the perturbation stands for planting of the species, while $b_{k}<0$ stands for harvesting. Denote

$$
\gamma \triangleq \frac{\omega}{T}
$$

We suppose that conditions (A1)-(A4) always hold in this paper. By the basic theories of impulsive differential equations in $[1,5]$, system $(1.1),(1.2)$ has a unique solution $x(t)=x\left(t, x_{0}\right) \in P C[R, R]$ for each initial value $x(0)=x_{0} \in R_{+}$and further $x(t)>0, t \in R_{+}$if $x(0)=x_{0}>0$ because of $1+b_{k}>0$ in (A4).

As a general single-species impulsive system, system (1.1), (1.2) includes many basic impulsive systems such as the logistic system studied by Liu and Chen [9]:

$$
\begin{gathered}
x^{\prime}(t)=x(t)(r(t)-a(t) x(t)), \quad t \neq \tau_{k}, \quad k \in N, \\
\triangle x\left(\tau_{k}\right)=b_{k} x\left(\tau_{k}\right), \quad k \in N,
\end{gathered}
$$


all the non-delay cases of single-species Lotka-Volterra system in [15], the model of respiratory dynamics in [6], the "food-limited" population growth model and the Michaelis-Menton single species growth model listed in [7] with $\gamma=1$, i.e., the periods of environment changing and impulsive perturbations are the same.

When $\gamma$ is rational, [9] showed that system (1.5), (1.6) has a unique positive periodic solution, which is a global attractor of all positive solutions if the following condition holds.

$$
\mu=\prod_{0<\tau_{k}<T}\left(\frac{1}{1+b_{k}}\right)^{\gamma} e^{-\int_{0}^{\omega} r(\tau) d \tau}<1 .
$$

And if (1.7) is reversed, then the zero solution is a global attractor. When $\gamma$ is irrational, system (1.5), (1.6) has no periodic solutions. [9] established sufficient conditions for the positive solutions of system (1.5), (1.6) attracting each other and suggested that system (1.5), (1.6) has a positive global attractor which is not periodic. This is quite different from the corresponding continuous system. However, to guarantee the existence of a positive global attractor, permanence should be established. Permanence is also biologically important since it demonstrates the stability of the system from the biological point of view and means that the density or the number of individuals of species is ultimately bounded both below and above by some positive constants independent of initial values.

The purpose of this paper is to establish permanence conditions for the general impulsive system (1.1), (1.2) and then as an application show that system (1.5), (1.6) is permanent with condition (1.7). This ensures the existence result of a global attractor suggested by Conjecture 3 in [9].

\section{Permanence}

We first give the definition of permanence.

DeFinition 2.1. System (1.1), (1.2) is called permanent iff there exist positive constants $M>\delta$ which are independent of initial values, such that any positive solution $x(t)$ of system (1.1), (1.2) satisfies

$$
\delta \leq \liminf _{t \rightarrow \infty} x(t) \leq \limsup _{t \rightarrow \infty} x(t) \leq M .
$$

From Definition 2.1, we can see that permanence means that each positive solution is ultimately bounded both above and below by some positive constants independent of the initial values of solution.

We now establish the ultimate lower bound of system (1.1), (1.2). If we express $n T, n \in N$, by making use of $\omega$, the following lemma is obviously valid.

Lemma 2.1. For any $n \in N, n T$ can be expressed by $\omega$ as

$$
n T=q_{n} \omega+s_{n},
$$

where $q_{n} \in N \cup\{0\}, s_{n} \in R_{+}, 0 \leq s_{n}<\omega$. Moreover, $\lim _{n \rightarrow \infty} q_{n}=\infty$ and $\lim _{n \rightarrow \infty} n / q_{n}=\gamma$. 
REMARK 2.1. If there exists $n_{0} \in N$ such that $s_{n_{0}}=0$, then $\gamma$ is rational. And if $s_{n}>0$ for any $n \in N$, then $\gamma$ is irrational.

THEOREM 2.1. If

$$
\mu=\prod_{0<\tau_{k}<T}\left(\frac{1}{1+b_{k}}\right)^{\gamma} e^{-\int_{0}^{\omega} f(\tau, 0) d \tau}<1
$$

then there exists a $\delta>0$ such that

$$
\liminf _{t \rightarrow \infty} x(t) \geq \delta
$$

where $x(t)$ is any solution of system (1.1), (1.2) with initial value $x(0)=x_{0}>0$.

Proof. By (2.1) the continuity of $f(t, \cdot)$ and the Lebesgue Theorem, we can choose $\delta_{1}>0$ be sufficiently small such that

$$
\prod_{0<\tau_{k}<T}\left(1+b_{k}\right)^{\gamma} e^{\int_{0}^{\omega} f\left(\tau, \delta_{1}\right) d \tau}>1
$$

As a consequence, by Lemma 2.1, there exist sufficiently small $\theta>0$ and sufficiently large $n_{0} \in N$ such that

$$
\prod_{0<\tau_{k}<T}\left(1+b_{k}\right)^{n / q_{n}} e^{\int_{0}^{\omega} f\left(\tau, \delta_{1}\right) d \tau}>1+\theta
$$

for $n \geq n_{0}$. Denote

$$
h=\min \left\{0, \min _{\tau \in[0, \omega]}\left\{f\left(\tau, \delta_{1}\right)\right\}\right\}, \quad H=\max \left\{0, \max _{\tau \in[0, \omega]}\left\{f\left(\tau, \delta_{1}\right)\right\}\right\} .
$$

Thus $h \leq 0 \leq H$. We will prove the result as the following two steps. We may suppose that $x_{0} \leq \delta_{1}$ since Step 1 can be skipped if $x_{0}>\delta_{1}$.

Step 1. There exists a $t_{0}>0$ such that $x\left(t_{0}\right)>\delta_{1}$.

Suppose on the contrary that

$$
x(t) \leq \delta_{1},
$$

for all $t \geq 0$. Then by (1.1) and (A3), we have

$$
x^{\prime}(t) \geq x(t) f\left(t, \delta_{1}\right), \quad t \geq 0, \quad t \neq \tau_{k}, \quad k \in N .
$$


By the comparison result of scalar impulsive differential equations [1, 5], Lemma 2.1 and (2.2), we find that

$$
\begin{aligned}
x(n T) & \geq x_{0} \prod_{0<\tau_{k}<n T}\left(1+b_{k}\right) e^{\int_{0}^{n T} f\left(\tau, \delta_{1}\right) d \tau} \\
& =x_{0} \prod_{0<\tau_{k}<n T}\left(1+b_{k}\right) e^{\int_{0}^{q_{n} \omega} f\left(\tau, \delta_{1}\right) d \tau} e^{\int_{q_{n} \omega}^{q_{n} \omega+s_{n}} f\left(\tau, \delta_{1}\right) d \tau} \\
& =x_{0} \prod_{0<\tau_{k}<T}\left(1+b_{k}\right)^{n} e^{q_{n} \int_{0}^{\omega} f\left(\tau, \delta_{1}\right) d \tau} e^{\int_{0}^{s_{n}} f\left(\tau, \delta_{1}\right) d \tau} \\
& =x_{0}\left(\prod_{0<\tau_{k}<T}\left(1+b_{k}\right)^{\frac{n}{q_{n}}} e^{\int_{0}^{\omega} f\left(\tau, \delta_{1}\right) d \tau}\right)^{q_{n}} e^{\int_{0}^{s_{n}} f\left(\tau, \delta_{1}\right) d \tau} \\
& \geq x_{0} e^{h \omega}(1+\theta)^{q_{n}},
\end{aligned}
$$

for $n \geq n_{0}$. Hence $x(n T) \rightarrow \infty$ as $n \rightarrow \infty$, which is a contradiction. Thus there exists a $t_{0}>0$ such that $x\left(t_{0}\right)>\delta_{1}$.

Step 2. Establish a positive ultimate lower bound $\delta \leq \delta_{1}$.

Let $t_{0}>0$ such that $x\left(t_{0}\right)>\delta_{1}$. If $x(t) \geq \delta_{1}$ for all $t \geq t_{0}$, then our aim is obtained for any positive constant $\delta \leq \delta_{1}$. We shall consider those solutions which leave region $\left\{x \mid x \leq \delta_{1}\right\}$ and reenter it. Let $t_{1}=\inf \left\{t>t_{0} \mid x(t) \leq \delta_{1}\right\}$. Then $x(t)>\delta_{1}, t \in\left[t_{0}, t_{1}\right)$ and $x\left(t_{1}\right) \geq \delta_{1}$. Suppose that $t_{1} \in\left(n_{1} T,\left(n_{1}+1\right) T\right]$ for some $n_{1} \in N \cup\{0\}$. Let $b=\min \left\{\prod_{t \leq \tau_{k} \leq T}\left(1+b_{k}\right) \mid t \in[0, T]\right\}$. By (2.1), as (2.2), we can choose an $m \in N, m>\gamma$ such that

$$
\prod_{0<\tau_{k}<T}\left(1+b_{k}\right)^{\gamma /(1+\gamma / m)} e^{\int_{0}^{\omega} f\left(\tau, \delta_{1}\right) d \tau}>1+\theta
$$

and

$$
b e^{h(\omega+T)-H \omega}(1+\theta)^{m / \gamma-1}>1 .
$$

Denote $n_{2}=\left(n_{1}+1+m\right)$. By Lemma 2.1, we have

$$
\frac{n_{2}-n_{1}-1}{q_{n_{2}}-q_{n_{1}+1}}=\frac{m \omega}{m T+s_{n_{1}+1}-s_{n_{2}}} \geq \frac{m \omega}{m T+\omega}=\frac{\gamma}{1+\gamma / m}
$$

and

$$
q_{n_{2}}-q_{n_{1}+1}=\frac{1}{\omega}\left(\left(n_{2}-n_{1}-1\right) T+s_{n_{1}+1}-s_{n_{2}}\right) \geq \frac{1}{\omega}(m T-\omega)=\frac{m}{\gamma}-1 .
$$


We claim that there must exist $t_{2} \in\left(t_{1}, n_{2} T\right]$ such that $x\left(t_{2}\right)>\delta_{1}$. Otherwise, $x(t) \leq \delta_{1}$ for $t \in\left(t_{1}, n_{2} T\right]$. Thus by

$$
x^{\prime}(t) \geq x(t) f\left(t, \delta_{1}\right), \quad t \in\left(t_{1}, n_{2} T\right], \quad t \neq \tau_{k}, \quad k \in N,
$$

we have

$$
\begin{aligned}
x & \left.n_{2} T\right) \\
\geq & x\left(t_{1}\right) \prod_{t_{1} \leq \tau_{k}<n_{2} T}\left(1+b_{k}\right) e^{\int_{t_{1}}^{n_{2} T} f\left(\tau, \delta_{1}\right) d \tau} \\
= & x\left(t_{1}\right) \prod_{t_{1} \leq \tau_{k} \leq\left(n_{1}+1\right) T}\left(1+b_{k}\right) \prod_{\left(n_{1}+1\right) T<\tau_{k}<n_{2} T}\left(1+b_{k}\right) \\
& \times e^{\int_{t_{1}}^{\left(n_{1}+1\right) T} f\left(\tau, \delta_{1}\right) d \tau} e^{\int_{q_{n_{1}}+1}^{q_{n_{2}} \omega} f\left(\tau, \delta_{1}\right) d \tau} \\
& \times e^{-\int_{q_{n_{1}+1} \omega}^{q_{n_{1}+1} \omega+s_{n_{1}+1}} f\left(\tau, \delta_{1}\right) d \tau} e^{\int_{q_{n_{2}} \omega}^{q_{n_{2}} \omega+s_{n_{2}} f\left(\tau, \delta_{1}\right) d \tau}} \\
\geq & \delta_{1} b e^{h(\omega+T)-H \omega}\left(\prod_{0<\tau_{k}<T}\left(1+b_{k}\right)^{\left(n_{2}-n_{1}-1\right) /\left(q_{n_{2}}-q_{n_{1}+1}\right)} e^{\int_{0}^{\omega} f\left(\tau, \delta_{1}\right) d \tau}\right)^{q_{n_{2}}-q_{n_{1}+1}} \\
\geq & \delta_{1} b e^{h(\omega+T)-H \omega}\left(\prod_{0<\tau_{k}<T}\left(1+b_{k}\right)^{\gamma(1+\gamma / m)} e^{\int_{0}^{\omega} f\left(\tau, \delta_{1}\right) d \tau}\right)^{q_{n_{2}}-q_{n_{1}+1}} \\
\geq & \delta_{1} b e^{h(\omega+T)-H \omega}(1+\theta)^{q_{n_{2}}-q_{n_{1}+1}} \\
\geq & \delta_{1} b e^{h(\omega+T)-H \omega}(1+\theta)^{m / \gamma-1} \\
> & \delta_{1}
\end{aligned}
$$

which is a contradiction. Thus there exists $t_{2} \in\left(t_{1}, n_{2} T\right]$ such that $x\left(t_{2}\right)>\delta_{1}$. Let $t_{3}=\inf \left\{t>t_{1} \mid x(t)>\delta_{1}\right\}$. Then $x(t) \leq \delta_{1}$ for $t \in\left(t_{1}, t_{3}\right]$.

Let $b_{1}=\min \left\{\prod_{t_{1} \leq \tau_{k}<t_{2}}\left(1+b_{k}\right) \mid 0<t_{1}<t_{2} \leq(m+1) T\right\}$ and $\delta=$ $\min \left\{\delta_{1}, \delta_{1} b_{1} e^{h(m+1) T}\right\}$. Obviously, $\delta$ is independent of any positive solution. Note that $x\left(t_{1}\right) \geq \delta_{1}$, we have for any $t \in\left(t_{1}, t_{3}\right]$,

$$
x^{\prime}(t) \geq x(t) f\left(t, \delta_{1}\right), \quad t \neq \tau_{k}, \quad k \in N
$$

and

$$
\begin{aligned}
x(t) & \geq x\left(t_{1}\right) \prod_{t_{1} \leq \tau_{k}<t}\left(1+b_{k}\right) e^{\int_{t_{1}}^{t} f\left(\tau, \delta_{1}\right) d \tau} \\
& \geq \delta_{1} b_{1} e^{h\left(t-t_{1}\right)} \\
& \geq \delta_{1} b_{1} e^{h(m+1) T} \\
& \geq \delta .
\end{aligned}
$$

Since $x\left(t_{3}^{+}\right)>\delta_{1}$, the same argument can be continued. We can conclude that $x(t) \geq \delta$ for all $t \geq t_{0}$. The proof is complete. 
REMARK 2.2. (2.1) can be rewritten as $\prod_{0<\tau_{k}<T}\left(1+b_{k}\right)^{\gamma} e^{\int_{0}^{\omega} f(\tau, 0) d \tau}>1$. By the proof of Theorem 2.1, we can see that it means biologically that when the quantity of the species is sufficiently small, then incorporating with the impulsive perturbations and the the intrinsic growth of the species, the average per capital increasing rate in one natural cycle is greater than 1.

Next, we establish the ultimate upper bound of system (1.1), (1.2). Suppose that

$$
\lim _{M \rightarrow \infty} \int_{0}^{\omega} f(\tau, M) d \tau=-\infty .
$$

Then we can choose $M_{1}>0$ be sufficient large such that

$$
\mu_{1}=\prod_{0<\tau_{k}<T}\left(1+b_{k}\right)^{\gamma} e^{\int_{0}^{\omega} f\left(\tau, M_{1}\right) d \tau}<1 .
$$

Using (2.4), the ultimate upper bound can be established by the method similar to the proof of Theorem 2.1. In fact, in Step 1, we can prove that there exists $t_{0}>0$ such that $x\left(t_{0}\right)<M_{1}$. Otherwise, we will lead to a contradiction, $x(n T) \rightarrow 0$ instead of $x(n T) \rightarrow \infty$ in the proof of Theorem 2.1. And then in Step 2, we can establish ultimate upper bound $M$ similarly. Hence we have the following theorem. Its proof will be omitted.

TheOREM 2.2. Suppose that (2.3) holds. Then there exists a constant $M>0$ such that $x(t) \leq M$ for $t$ sufficiently large, where $x(t)$ is any solution of system (1.1), (1.2) with $x(0)=x_{0}>0$.

Theorems 2.1 and 2.2 establish the permanence of system (1.1), (1.2).

Theorem 2.3. Suppose that (2.1) and (2.3) hold. Then system (1.1), (1.2) is permanent.

\section{Application}

The permanence conditions (2.1) and (2.3) give some simple conditions on the parameters of system (1.1), (1.2). Thus our results can be directly applied to the special cases of basic single species impulsive systems such as the logistic system (1.5), (1.6) in [9], all the non-delay cases of single-species Lotka-Volterra system in [15], the model of respiratory dynamics in [6], the "food-limited" population growth model and the Michaelis-Menton single species growth model listed in [7].

To illustrate it clearly, we now consider system (1.5), (1.6) as an example. Suppose that (1.5) is $\omega$-periodic, (1.6) is $T$-periodic, i.e.,

$$
r(t+\omega) \equiv r(t), \quad a(t+\omega) \equiv a(t), \quad t \in R,
$$


$T$ is the least positive constant such that there are $l \tau_{k} \mathrm{~S}$ in the interval $(0, T)$ with

$$
\tau_{k+l}=\tau_{k}+T, \quad b_{k+l}=b_{k}, \quad k \in N
$$

and further

$$
r(\cdot), a(\cdot) \in P C[R, R] .
$$

The following restrictions on system (1.5), (1.6) are natural from the biological meanings.

$$
\begin{gathered}
a(t) \geq 0, \quad t \in R_{+}, \\
1+b_{k}>0, \quad b_{k} \neq 0, \quad k \in N .
\end{gathered}
$$

We suppose that (3.1)-(3.5) are satisfied basically by system (1.5), (1.6). Then obviously, (A1)-(A4) hold. Using Theorem 2.3, we have the following result.

TheOREM 3.1. Suppose that (1.7) and

$$
\int_{0}^{\omega} a(\tau) d \tau>0
$$

hold. Then system (1.5), (1.6) is permanent.

Proof. Condition (1.7) is exactly (2.1) for system (1.5), (1.6). By (3.6), (2.3) obviously holds. Thus the result follows directly from Theorem 2.3. The proof is complete.

The ultimate upper bound of system (1.5), (1.6) is established in [9] by the method of Lyapunov function, which relies on

$$
r(t)>0, \quad a(t)>0, \quad t \in R_{+} .
$$

Condition (3.7) means that the birth rate is always larger than death rate and the density dependance always exists. It may be unreasonable for some species living in a periodic changing environment, for example, birth may take place seasonally. In this paper, condition (3.7) is replaced by (3.4) and (3.6), which only contain restrictions for $a(t)$. In fact, condition (1.7) already has restrictions for $r(t)$. We can see clearly that there are no positive restrictions for $r(t)$ and $a(t): r(t)$ may be negative for some $t$, which is reasonable for species with seasonal birth and only $a(t) \geq 0$ is necessary. Hence the conditions and results are quite reasonable. Even when $r(t)<0$ for all $t>0$, which is reasonable for endangered species, Theorem 3.1 suggests that the species could be permanent if $b_{k}$ and $T$ are large enough. In this sense, impulsive planting is helpful for protecting the species from extinction.

When $\gamma$ is rational, based on (3.7), [9] proved that if (1.7) holds, system (1.5), (1.6) has a unique positive global attractor which is a positive periodic solution. The system is then obviously permanent. Theorem 3.1 shows that with this condition 
and condition (3.6), system (1.5), (1.6) is permanent whether $\gamma$ is rational or not. Hence Theorem 3.1 ensures the existence of a positive global attractor suggested in [9, Conjecture 3]. With condition (1.7), [9] proved that the positive solutions of system (1.5), (1.6) attracts each other in the sense of lower limit. Thus the permanence result in this paper also strongly suggests that the global attractivity results in [9, Conjectures 1 and 2] are valid. Since the positive global attractor of system (1.5), (1.6) is not periodic, which is different from the corresponding continuous system, it is interesting to study further its structure.

Acknowledgement. We thank the referee and Prof. M. Mimura for their suggestions that improved the presentation of this paper.

\section{References}

[ 1 ] D.D. Bainov and P. S. Simeonov, Impulsive Differential Equations: Periodic Solutions and Applications. Longman, England, 1993.

[ 2 ] E. Funasaki and M. Kot, Invasion and chaos in a periodically pulsed mass-action chemostat. Theor. Popul. Biol., 44 (1993), 203-224.

[ 3 ] S. Gao and L. Chen, The effect of seasonal harvesting on a single-species discrete population model with stage structure and birth pulses. Chaos, Solitons \& Fractals, 24 (2005), 1013-1023.

[ 4 ] A. Lakmeche, O. Arino, Birfurcation of non trivial periodic solutions of impulsive differential equations arising chemotherapeutic treatment. Dynam. Contin. Discrete Impuls., 7 (2000), 265-287.

[ 5 ] V. Lakshmikantham, D.D. Bainov and P.S. Simeonov, Theory of Impulsive Differential Equations. World Scientific, Singapore, 1989.

[6] W. Li and H. Huo, Global attractivity of positive periodic solutions for an impulsive delay periodic model of respiratory dynamics, J. Comput. Appl. Math., 174 (2005), 227-238.

[ 7 ] W. Li and H. Huo, Existence and global attractivity of positive periodic solutions of functional differential equations with impulses. Nonlinear Analysis, 59 (2004), 857-877.

[ 8 ] B. Liu, Y. Zhang and L. Chen, The dynamical behaviors of a Lotka-Volterra predator-prey model concerning integrated pest management. Nonlinear Anal. Real World Appl., 6 (2005), 227-243.

[ 9 ] X. Liu and L. Chen, Global dynamics of the periodic logistic system with periodic impulsive perturbations. J. Math. Anal. Appl., 289 (2004), 279-291.

[10] X. Liu and L. Chen, Global attractivity of positive periodic solutions for nonlinear impulsive systems. Nonlinear Analysis, 65 (2006), 1843-1857.

[11] M.G. Roberts and R.R. Kao, The dynamics of an infectious disease in a population with birth pulses. Math. Biosci., 149 (1998), 23-36.

[12] B. Shulgin, L. Stone and Z. Agur, Pulse vaccination strategy in the SIR epidemic model. Bull. Math. Biol., 60 (1998), 1123-1148.

[13] S. Tang and L. Chen, Density-dependent birth rate, birth pulses and their population dynamic consequences. J. Math. Biol., 44 (2002), 185-199.

[14] S. Tang and L. Chen, Global attractivity in a "food-limited" population model with impulsive effects. J. Math. Anal. Appl., 292 (2004), 211-221.

[15] J. Yan, A. Zhao and J.J. Nieto, Existence and global attractivity of positive periodic solution of periodic single-species impulsive Lotka-Volterra systems. Math. Comput. Model., 40 (2004), 509-518.

[16] B. Zhang and Y. Liu, Global attractivity for certain impulsive delay differential equations. Nonlinear Analysis, 52 (2003), 725-736.

[17] W. Zhu, D. Xu and Z. Yang, Global exponential stability of impulsive delay difference equation. Appl. Math. Comput., 181 (2006), 65-72. 
